\title{
Improving quality of life in patients with end-stage age-related macular degeneration: focus on miniature ocular implants
}

REVIEW

This article was published in the following Dove Press journal:

Clinical Ophthalmology

29 December 2011

Number of times this article has been viewed

Michael A Singer'

Nancy Amir ${ }^{2}$

Angela Herro ${ }^{3}$

Salman S Porbandarwalla ${ }^{3}$

Joseph Pollard'

'Medical Center Ophthalmology Associates, San Antonio, TX, USA;

${ }^{2}$ Santa Rosa Low Vision Clinic, San

Antonio, TX, USA; ${ }^{3}$ University of Texas Health Science Center at San Antonio, San Antonio, TX, USA

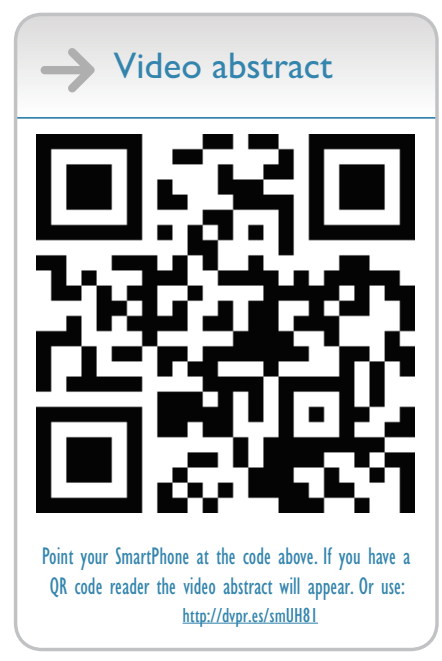

Correspondence: Michael A Singer 9157 Huebner Road, San Antonio,

TX, 78240, USA

Tel +I 2106972020

Fax + I 2105587687

Email msinger@mcoaeyecare.com

\begin{abstract}
Low vision devices in the past have been mainly extraocular. There are now four new devices in different stages of development and implementation that are currently available. Three of them, the Implantable Miniature Telescope (IMT, VisionCare Ophthalmic Technologies, Saratoga, CA), Intraocular Lens for Visually Impaired People (IOL-VIP, IOL-VIP System, Soleko, Pontecorvo, Italy), and Lipschitz Mirror Implant (LMI, Optolight Vision Technology, Herzlia, Israel) are implanted into the anterior segment while the Argus II (Second Sight Medical Products, Sylmar, CA) is implanted into the posterior segment. The goal of these devices is to increase the patient quality of life which has been measured by Visual Functioning Questionnaire (VFQ) scales. The IMT is the only device that has been shown to increase the VFQ score by seven points at 6 months compared to baseline. It is the only FDA-approved device in the US while the Argus has been approved in Europe. Each of these prosthetics has potential benefits for patients.
\end{abstract}

Keywords: IMT, IOL-VIP, LMI, Argus II

\section{Introduction}

Age-related macular degeneration (AMD) is the leading cause of blindness in adults over the age of 65 years. ${ }^{1}$ As the life expectancy in civilized countries increases, the growing incidence of AMD presents an important public health concern. ${ }^{2}$ Dry AMD accounts for approximately $90 \%$ of all AMD cases and is characterized by the accumulation of deposits in Bruch's membrane and pigmentary changes in the retinal pigment epithelium. Wet AMD is the more severe form, accounts for $10 \%$ of all AMD cases and is caused by retinal tissue invasion of new blood vessels, choroidal neovascularization $(\mathrm{CNV}) .{ }^{3}$ The macula is the region of the retina that provides central vision. CNV leads to inflammation, edema and scarring, with loss of structure and function of the macula.

Treatment for dry AMD has primarily focused on preventing at-risk patients from progressing to the wet form. Treatment for wet AMD has been limited. ${ }^{4}$ The age-related changes that cause $\mathrm{CNV}$ are not completely understood, but vascular endothelial growth factor (VEGF) has been identified as an important factor promoting neovascularization. ${ }^{5}$ Treatment for AMD includes administration of anti-VEGF. Ranibizumab (Lucentis ${ }^{\circledR}$, Genentech, San Francisco, CA) was approved by the Food and Drug Administration for the treatment of all angiographic subtypes of subfoveal neovascular age-related macular degeneration in $2006 .{ }^{6}$ Ranibizumab has been shown to improve visual acuity by three lines in $30 \%-40 \%$ of patients with monthly dosing. ${ }^{7}$ Bevacizumab has been shown to be equivalent to Ranibizumab with monthly dosing. ${ }^{8}$ 
However, anti-VEGF treatment does not work for everyone. Despite treatment some patients still progress, especially those with geographic atrophy. ${ }^{9}$

Advanced AMD, a common pathway of both the dry and wet form, is the end-stage with presentation of bilateral, untreatable macular scars causing central scotomata (blind spots). These blind spots result in significant visual impairment that limits a patient's daily activities requiring good central vision, such as self-care, reading, recognizing facial features, driving, watching television, and other social activities. Advanced AMD is also associated with legal blindness, increased risk of depression, increased levels of dependency, increased risk of accidents, and a significant decrease in quality of life (QOL). ${ }^{10-12}$ Currently, there are no medical treatments for end-stage AMD.

\section{Extraocular low vision aids}

Visual rehabilitation with low vision magnifiers has been the mainstay of treatment for patients with advanced dry macular degeneration, diabetic retinopathy, and retinal vein occlusions. ${ }^{13}$ Patients with these diseases have a decreased ability to carry out activities of daily living including reading, writing, and grooming which in turn causes a deterioration in their QOL.

Currently there are many nonsurgical options for visual rehabilitation, some examples are: hand/stand magnifiers, spectacles, hand held telescopes, closed circuit televisions, and high plus spectacles in conjunction with high minus contact lenses to create a telescopic effect. Although these tools maybe effective for correcting overall visual functioning, there are several limitations especially when correcting distance and near acuity.

In order to obtain effective magnification, patients have to bring objects closer to the eye to increase the angle of resolution. In addition to moving objects closer, these devices are cumbersome to use, they are cosmetically burdensome, and most of all, these external devices restrict effective field of view. The majority of these magnifying devices have a restricted field-of-view and have to be manually scanned across the text, magnifying only a small portion at a time. In all cases, the scanning is slow and limits the usefulness of the device. Patients are also required to use head movements or hand movements more often than using natural eye movements to scan which results in vestibular-ocular conflict and motion sickness. Furthermore, patients afflicted with arthritis, Parkinson's disease or other neuromuscular disorders, or dementia, among others, will have difficulty maneuvering these devices in a useful way. Despite all this, patients are willing to try anything for a chance of better vision, no matter how difficult and burdensome these devices may be.

In addition to the hand-held and stand magnifiers, spectacle-mounted telescopes are a great alternative. They not only provide good magnification but also allow using eye movements to a limited field of fixation. The drawback of these devices is that they are very dependent on vertex distance. For example, Nguyen et al reported fields from $5^{\circ}$ to $11^{\circ}$ for varying telescopes set at $10 \mathrm{~mm}$ from the cornea. ${ }^{14}$ Bailey reported for a $3.0 \times$ spectacle-mounted telescope a field-of-view of $11^{\circ}$ for the Galilean design and $14^{\circ}$ for the Keplerian design. ${ }^{15}$ With such a narrow field, navigation in the visual environment is difficult and may be dangerous. Patients are forced to use head scanning to cover a wider angle through the telescope.

The limitations on the field-of-fixation are even more severe with the combined IOL/spectacle telescopic (or contact lens/spectacle) system. With this device, the field-of-fixation was computed to be only between $1^{\circ}$ and $5^{\circ}$ depending on lens design and vertex distance. ${ }^{14}$

For distance vision, the choice is limited to telescopes, headset devices, and closed circuit televisions, all of which have a high cost/benefit ratio and generally lead to patient frustration and abandoning of the device. ${ }^{15}$ However, for many years, low-vision magnifiers have provided patients with an opportunity to enhance their existing vision with the goal to improving their QOL. Furthermore, this was an attractive option as a noninvasive way for patients to avoid surgery and have the opportunity to gain vision and until recently, this was the only option available. Current research and development are aiming at ways to utilize the natural eye and visual system to augment the parts of the patient's architecture with normal function. The future of low magnifiers now includes devices that can be implanted in the eye, improving the field of magnification and ease of use.

\section{Intraocular implants}

Intraocular implants are an attractive and intuitive way to circumvent many of the problems faced in extraocular visual aids. With the addition of plus lenses, these devices may be used for near activities as well. There are currently four different types of intraocular implant available for use and these include both devices placed in the anterior segment as well as the posterior segments of the eye. We will review three devices for the anterior segment, the Implantable Miniature Telescope (IMT by Dr Isaac Lipshitz, VisionCare Ophthalmic Technologies, Saratoga, CA), ${ }^{4,13-21}$ the IOL-VIP system (IOL-VIP System, Soleko, Pontecorvo, Italy), ${ }^{16}$ and the 
Lipschitz Mirror Implant (LMI, Optolight Vision Technology, Herzlia, Israel), ${ }^{22}$ and one device for the posterior segment, the Argus II retinal implant by Second Sight (Second Sight Medical Products, Sylmar, CA). ${ }^{23}$ There are many advantages to such implants as compared to magnifiers and spectacles as described previously. One very important consideration is eye movement control with the field of view. Because scanning eye movements are necessary for normal functioning, it is easy to imagine why magnifiers or spectacles could impede the best sight as the patient would have to be constantly moving the lens to a new point in space as well as adjust to the interaction of the ring scotoma caused by such devices. With the device implanted within the eye, many of these barriers are eliminated. Furthermore, the effective field-of-fixation varies based on design where a Galilean design affords approximated $6^{\circ}$ for the $3.0 \times$ design and $10^{\circ}$ for the Keplerian design. Furthermore, with the combined IOL/spectacle device, the computed field is between $1^{\circ}$ and $5^{\circ}$, dramatically less than the $20^{\circ}$ for the $3 \times$ and $24^{\circ}$ with the $2.2 \times$ IMT devices.

A second important characteristic of implantable devices is the improvement in head motion and vestibular effects. Whenever using a head-mounted magnifying device, there are disruptions in image stability and image direction. Furthermore, with the vestibular ocular reflex (VOR), eye movements are programmed to occur one-to-one with head movement. However, when using a magnifier with $3.0 \times$ magnification for example, an eye movement three times as large as head rotation is required which is very difficult if not impossible to achieve by VOR adaptation. This is further complicated when using a telescope monocularly as there is a different demand between the two eyes. This problem is resolved by the different implantable systems since a given head movement requires a compensatory eye movement of the same magnitude.

A third advantage of intraocular devices is the improvement in monocular depth perception. Any device that is using a monocular magnification will have to overcome the intrinsic problem of altered depth perception because of the lack of binocular disparity and stereovision. Oftentimes the use of monocular cues such as interposition, relative sizes of known objects, linear perspective, foreshortening and aerial perspective, can provide a useful sense of depth perception. Another phenomenon important in monocular depth perception is motion parallax, where objects closer to the eye appear to move faster than objects moving at the same speed at a farther distance. This same phenomenon can be used to judge depth in static objects by the lateral movement of the eye or moving the head so that a closer object will move faster on the retina and cover a larger area than a farther object. This cannot usually be accomplished by rotational eye movements because the rotational axis is close to the nodal point however the nodal point of the eye is shifted anteriorly by a telescope system and thus can generate enough parallax to provide fine depth perception for near tasks.

\section{Anterior segment devices}

The concept of using a minus and plus lens as a magnifier for retinal image enlargement has been used for visual rehabilitation in low-vision patients for many years. Implants like the IMT and the IOL-VIP system have been shown to have promising results.

\section{IMT device}

The IMT is a visual prosthetic device that provides the eye with an enlarged retinal image of the central visual field with the goal of improving central vision in one eye of patients with moderate to profound visual impairment. ${ }^{13}$ Earlier generations of the IMT have been reported. ${ }^{19}$ It incorporates ultra-precision wide-angle micro-optics that, together with the cornea, functions as a fixed focus telephoto system. The housing measures $3.6 \mathrm{~mm}$ in diameter and $4.4 \mathrm{~mm}$ in length in a polymethylmethacrylate carrier with haptics measuring $13.5 \mathrm{~mm}$ in diameter designed to be implanted "in the bag" (Figure 1). In doing so, it generates an image over $55^{\circ}$ of the retina, versus the $5^{\circ}$ focused by the natural crystalline lens. Furthermore, this can be done while still minimizing the patient's scotoma and retaining a relatively wide $20^{\circ}-24^{\circ}$ field of view. The device has undergone Phase II and III

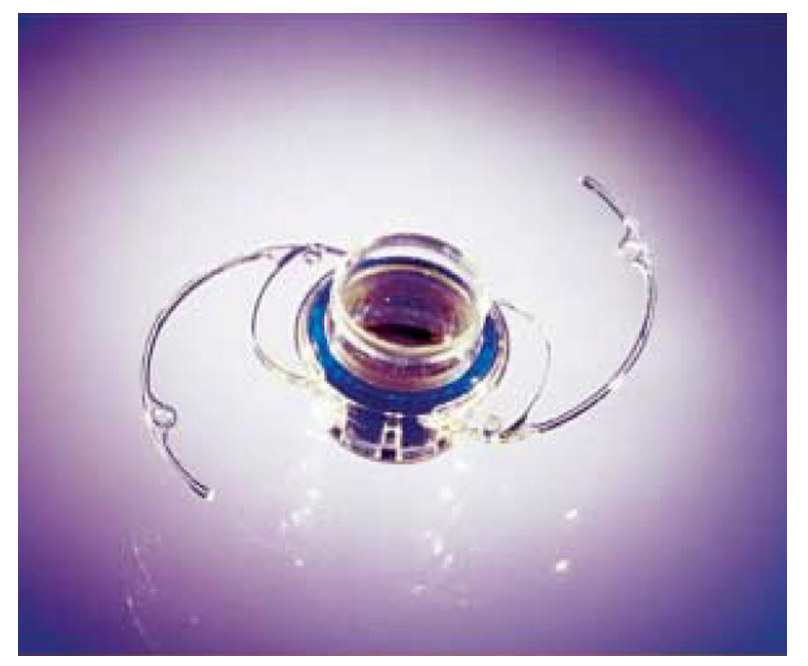

Figure I Implantable Miniature Telescope (IMT by Dr Isaaz Lipshitz; VisionCare Ophthalmic Technologies,Saratoga, CA, USA)..$^{18}$ 
testing and has very promising results and was been approved by the Food and Drug Administration in July of 2010. Most patients enrolled in the Phase III studies have had a baseline mean composite score of 44/100 on the National Eye Institute Visual Functioning Questionnaire [NEIVFQ]-25. Most had disciform scarring due to CNV or geographic atrophy and the mean endothelial cell count was 2492 cells $/ \mathrm{mm}^{2}$. Patients excluded were those with active CNV or treatment within the preceding 6 months, history of intraocular or corneal surgery, pathology that compromises peripheral vision in the fellow eye, endothelial cell density $<1600$ cells $/ \mathrm{mm}^{2}$, anterior chamber depth $<2.5 \mathrm{~mm}$, myopia or hyperopia $>6.0 \mathrm{D}$, glaucoma, or other retinal or optic nerve pathology. ${ }^{4}$

This device has the most peer-reviewed literature discussing its patient selection, use and outcomes, and is the only telescope system that is FDA approved. A specific surgical procedure has been described to minimize endothelial cell loss and morbidity associated with the implantation. Typically, the procedure is done beginning with a retrobulbar block and the lens is removed using extracapsular cataract extraction with phacoemulsification. The size of the device requires a $12-\mathrm{mm}$ partial thickness limbal incision and a capsulorhexis of at least $7 \mathrm{~mm}$ and is closed with 10.0 nylon sutures. After the ophthalmic viscoelastic device (OVD) is removed, the pupil is constricted using an intraocular miotic and a peripheral iridectomy is performed. ${ }^{18}$ Because of the size of the device relative to standard intraocular lenses, the corneal endothelial health raises a significant concern. There has been a documented decline of approximately $20 \%$ at 3 months postoperatively. However, there is a low rate of additional endothelial cell loss, leading to the assumption that most damage occurs at the time of surgery and can be minimized by careful attention to surgical technique. ${ }^{21}$

A potential risk related to the procedure is corneal endothelial cell loss that can affect overall corneal health. Longer-term FDA safety data collected for over 4 years follow-up showed 5/217 (2.3\%) of patients required a corneal transplant. ${ }^{24}$ Mean endothelial cell loss was $25 \%$ at 1 -year postoperative, and stabilized from 1 through 2 years, with $2.4 \%$ mean cell loss occurring in that period. There was no significant change in coefficient of variation or percentage of hexagonal endothelial cells from within 6 months to 2 years after surgery. The most common complication was inflammatory deposits. ${ }^{25}$

The procedure is very safe and has very few anterior segment complications in long term follow-up. This has led investigators to achieve results comparable to current standards of care in rates of keratopathy and postoperative complications, keeping in mind the relatively short follow-up.

The IMT has had very promising success in clinical trials. The primary efficacy end-point required by the FDA protocol was to achieve greater than or equal to $50 \%$ of patients gaining two or more lines of either near or distance visual acuity at 12 months. At the 12-month mark, this end-point was achieved in $90 \%$ of patients with $67 \%$ gaining three or more lines in best-corrected distance visual acuity. A secondary end-point was improvement in QOL as measured by the NEIVFQ-25 which showed mean NEI VFQ-25 scores at 1 year improved by more than 7 points from baseline $(P<0.01)$ on 7 of 8 relevant subscales. This telescope has the potential to afford a major advance in the vision and QOL to many patients with moderate to profound vision loss without the cumbersome extraocular visual aids or social stigma associated with hardware on and around the eye. ${ }^{4}$

\section{The IOL-VIP system}

The IOL-Vip System is a Lenspecial project from Soleko. It uses two IOLs that act as a Galilean telescope, a high minus IOL in the bag, and a high plus anterior chamber intraocular lens (ACIOL) (Figure 2). A clinical trial using the IOL-VIP system was carried out in 40 eyes of 35 patients. ${ }^{17}$ Extensive preoperative training and software recognition programs were used to select these 35 patients. All the surgeries were performed at an ambulatory surgery center (ASC), and patients underwent a laser PI before placement of the ACIOL

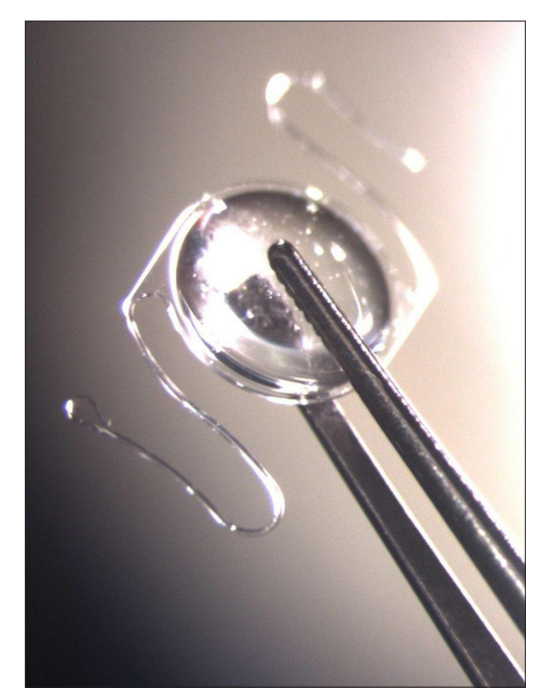

Figure 2 Intraocular Lens for Visually Impaired People (IOL-VIP System; Soleko, Pontecorvo, Italy). ${ }^{16}$ 
(either preoperative or intraoperative). In addition every patient had a preoperative endothelial cell count.

The study did not find a difference in complication or endothelial cell count when compared to the conventional phacoemulsification procedure. There were some complications including elevated IOP from pupillary block, anterior capsule fibrosis, and posterior capsular opacification, all of which were treated using the ND YAG laser. All of the patients completed the postoperative 3-month rehabilitation program. They found that all the 40 eyes (both groups - the severely low vision and the mild-low vision) had improvement in their BCVA postoperatively, however the mild-low vision group seemed to have a better outcome.

The IOL-VIP system allows patients to retain their peripheral vision. This IOL-VIP implant has been used in patients with central scotomas and maculopathy since 2002 in Spain and in the UK. ${ }^{16}$

Although this is an excellent alternative to the conventional external low-vision aids there might be some additional factors to take into consideration. Using two lenses, especially an ACIOL, can have a crowding effect leading to glaucoma or angle closure especially in hyperopic patients. Furthermore the concave and convex lenses that provide magnification are at the lens-aqueous interface, as these lenses are surrounded by aqueous. Thus, the magnification is limited to $1.3 \times .{ }^{15}$ In addition, the pre- and postoperative visual rehabilitation takes months, which could be inconvenient for patients. ${ }^{16}$

Overall, the IOL-VIP system seems to have a favorable outcome on the patients that currently have these implants and the results seem promising.

\section{Intraocular mirror telescopic intraocular lens (Lipshitz Mirror implant or LMI)}

The Lipshitz implant is a modified conventional IOL that follows the Cassegrain telescopic configuration, using two miniature mirrors. ${ }^{22}$ The IOL has a dual optical system by which light passing through the positive and negative lenses ends up being magnified (Figure 3 ). The LMI implant has been designed to give the patient $2.5 \times$ central retinal magnification. ${ }^{22}$

A prospective, nonrandomized pilot clinical trial was conducted at Dr Agarwal's Eye Research Center from June to December 2006. Six eyes of six patients were selected, with macular pathology and corrected VA $<20 / 200$ whose vision improved with a $2.5 \times$ external telescope.

Postoperatively, patients were tested for changes in central VA, and were asked to complete a QOL questionnaire. The

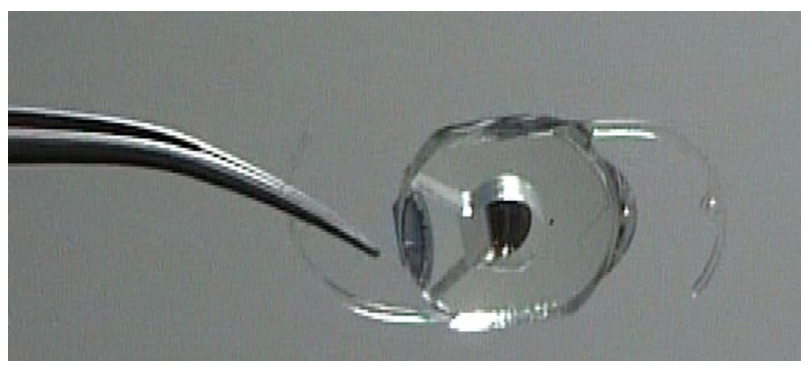

Figure 3 Lipshitz Mirror implant (LMI; Optolight Vision Technology, Herzlia, Israel). ${ }^{22}$

LMI implant was well tolerated in all the patients with no intra-operative complications. In addition, most patients reported an improvement in their central visual acuity and their QOL. Some patients complained of glare in the immediate postoperative period that seemed to be suppressed by the 6 -month follow-up. ${ }^{22}$

There is also a new model of the mirror telescope called the OriLens and it is for pseudophakic patients. It is a sulcus fixated lens placed over an existing IOL. It is indicated for phakic and pseudophakic eyes and is only $1.25 \mathrm{~mm}$ thick. It is currently the only option for pseudophakic patients. The OriLens is CE certified and is used in Europe and other nonFDA regulated markets.

\section{Retinal implants}

In contrast to using image magnification via optical devices to improve vision, a number of retinal-based prostheses are currently under investigation. These devices differ from the implantable prostheses in many ways. They are composed of highly complex integrated microchips and are offered to patients who are almost completely blind from retinal degenerations such as retinitis pigmentosa. The Argus II ${ }^{26,27}$ epiretinal prosthesis was shown to improve performance in spatial motor tasks, and the subretinal Retina Implant ${ }^{28}$ (Retina Implant AG, Tubingen-Reutlingen, Germany) was shown to allow blind patients to recognize images and read letters. However, the latter retinal implant is not applicable at present in patients with age-related macular degeneration and thus will not be discussed in this review. The Argus II is a retinal prosthesis system that combines a digital camera built into a pair of glasses, which processes the images into electrical impulses and transmits these pulses to a transmitter that communicates with the retinal implant stimulating the nerve cells and taking advantage of an intact optic nerve and posterior visual pathway (Figure 4). Currently, the implant consists of an array of 60 electrodes on a $1 \mathrm{~mm} \times 1 \mathrm{~mm}$ chip and can give subjects the ability to perceive shapes in contrast in relatively 
low resolution. Argus II recently received the CE mark and is now available for sale in Europe. The researchers at Second Sight are currently working on Argus III and IV which are projected to have as many as 1000 electrodes in an effort to gain facial recognition. Retinal implants provide another exciting advance in the treatment of profound vision loss.

\section{Discussion}

When comparing anterior segment implants, the IMT has significantly more peer-reviewed literature and clinical data than the IOL-VIP System or the Lipshitz Mirror IOL. The IOL-VIP system has been reviewed at one center with a relatively low number of eyes $(n=40)$. The magnification has been minimal $(1.3 \times)$ and a prismatic effect has been reported.

The initial study of the LMI involved a low number of eyes $(n=6)$ and a short follow-up time ( 6 months) at only one center. In a study by Agarwal et al, ${ }^{22}$ the advantages of this implant over the IMT are discussed, which include preservation of peripheral vision, no significant loss of endothelial cells as a result of an easier procedure, and finally the ability to place bilateral implants in patients, unlike the IMT. However, some patients noted the glare from the mirrored IOLs to be bothersome, especially from a cosmetic standpoint in younger patients.

The IMT has been FDA-approved for the treatment of AMD. When compared to the other anterior segment implants, the number of IMT patients is significantly higher in the 1-year $(n=217)$ and 2-year $(n=174)$ follow-up reports. Patients involved in the IMT clinical trial are currently in long-term follow-up and are approaching 96-month (8-year) follow-up visits.

The QOL improvements with the IMT, as mentioned previously, are of particular note. The NEIVFQ is considered the gold standard QOL measure for ophthalmic interventions. A five-point improvement in the VFQ-25 score is considered

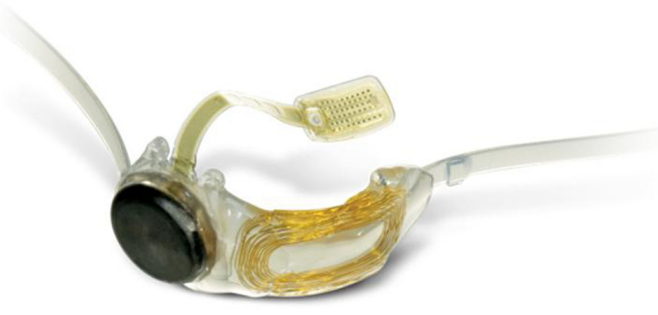

Figure 4 Argus II (Second Sight Medical Products, Sylmar, CA, USA). clinically meaningful: the 1-year IMT follow-up showed a clinically meaningful increase in the VFQ-25 when compared to baseline. The 1- and 2-year follow-up articles on the IMT show that improved vision as a result of the telescope-eye best corrected visual acuity is responsible for increased QOL in study subjects. Furthermore, a recent study has shown the procedure to be cost-effective in terms of the utility gained by patients. ${ }^{29}$

\section{Conclusion}

Low-vision aids and intraocular implants are all designed to help patients improve their vision in end-stage age-related macular degeneration and by extension, QOL. We have shown that the current direction should logically trend toward smaller, less cumbersome, more efficient and effective devices for this increasing patient population. This can best be accomplished through intraocular implants. In addition, these devices with the addition of plus lenses, have the ability to be used for both distance and near.

The anterior segment implants have the advantage of utilizing a surgical skill that has already been honed by many ophthalmologists in the placement of posterior chamber IOLs after cataract surgery. At this time, the only FDA-approved implant is the implantable miniature telescope (IMT) device for the US and the Argus II posterior segment implant for the European Union. These are promising achievements and a welcome addition to the existing armamentarium of lowvision devices. These devices help to increase the QOL in low-vision patients until a preventative intervention or cure is discovered.

\section{Disclosure}

The authors report no conflicts of interest in this work.

\section{References}

1. Friedman DS, O'Colmain BJ, Munoz B, et al; for the Eye Diseases Prevalence Research Group. Prevalence of age-related macular degeneration in the United States. Arch Ophthalmol. 2004;122(4): 564-572.

2. Gohdes DM, Balamurugan A, Larsen BA, Maylahn C. Age related eye diseases: an emerging challenge for public health professionals. Prev Chronic Dis. 2005;2(3):A17. Available from: http://www.cdc.gov/pcd/ issues/2005/jul/04_0121.htm. Accessed August 1, 2011.

3. AREDS Research Group. A randomized, placebo-controlled, clinical trial of high-dose supplementation with vitamins $\mathrm{C}$ and $\mathrm{E}, \beta$-carotene, and zinc for age-related macular degeneration and vision loss: AREDS report no. 9. Arch Ophthalmol. 2001;119(10):1439-1452.

4. Chun DW, Heier JS, Raizman MB. Visual Prosthetic Device for bilateral end-stage macular degeneration. Expert Rev Med Devices. 2005;2(6):657-665.

5. Lopez PF, Sippy BD, Lambert HM, Thach AB, Hinton DR. Transdifferentiated retinal pigment epithelial cells are immunoreactive for vascular endothelial growth factor in surgically excised age related macular degeneration-related choroidal neovascular membranes. Invest Ophthalmol Vis Sci. 1996;37(5):855-868. 
6. Rosenfeld PJ, Brown DM, Heier JS, et al; MARINA Study Group. Ranibizumab for neovascular age-related macular degeneration. $N$ Engl J Med. 2006;355(14):1419-1431.

7. Brown DM, Kaiser PK, Michels M, et al. Ranibizumab versus verteporfin for neovascular age-related macular degeneration. $N$ Engl J Med. 2006;355(14):1432-1444.

8. Martin DF, Maguire MG, Ying GS, et al; CATT Research Group. Ranibizumab and bevacizumab for neovascular age-related macular degeneration. N Engl J Med. 2011;364(20): 1897-1908.

9. Sunness JS. The natural history of geographic atrophy, the advanced atrophic form of age-related macular degeneration. Mol Vis. 1999;5:25.

10. Williams RA, Brody BL, Thomas RG, Kaplan RM, Brown SI. The psychosocial impact of macular degeneration. Arch Ophthalmol. 1998; 116(4):514-520.

11. Casten RJ, Rovner BW, Tasman W. Age-related macular degeneration and depression: a review of recent research. Curr Opin Ophthalmol. 2004;15(3):181-183.

12. Brown MM, Brown GC, Sharma S, Kistler J, Brown H. Utility values associated with blindness in an adult population. Br J Ophthalmol. 2001;85(3):327-331.

13. Peli E. The optical functional advantages of an intraocular low-vision telescope. Optom Vis Sci. 2002;79(4):225-233.

14. Nguyen A, Nguyen A-T, Hemenger RP, Williams DR. Resolution, field of view, and retinal illuminance of miniaturized bioptic telescopes and their clinical significance. $J$ Vis Rehab. 1993;7:5-9.

15. Bailey IL. Critical view of an ocular telephoto system. CLAO J. 1987; 13(4):217-221.

16. Orzalesi N, Pierrottet CO, Zenoni S, Savaresi C. The IOL-Vip System: a double intraocular lens implant for visual rehabilitation of patients with macular disease. Ophthalmology. 2007;114(5):860-865.

17. Hudson HL, Lane SS, Heier JS, et al; IMT-002 Study Group. Implantable miniature telescope for the treatment of visual acuity loss resulting from end-stage age-related macular degeneration: 1-year results. Ophthalmology. 2006;113(11):1987-2001.

18. Colby KA, Chang DF, Stulting RD, Lane SS. Surgical placement of an optical prosthetic device for end-stage macular degeneration: the implantable miniature telescope. Arch Ophthalmol. 2007;125(8): 1118-1121.
19. Hudson HL, Stulting RD, Heier JS, et al; IMT002 Study Group Implantable telescope for end-stage age-related macular degeneration: long-term visual acuity and safety outcomes. Am J Ophthalmol. 2008; 146(5):664-673.

20. Primo SA. Implantable miniature telescope: lessons learned. Optometry. 2010;81(2):86-93.

21. Bansal AS, Baker P, Haller JA. An implantable visual prosthetic for end-stage macular degeneration. Expert Rev Ophthalmol. 2011;6(2): $141-145$.

22. Agarwal A, Lipshitz I, Jacob S, et al. Mirror telescopic intraocular lens for age-related macular degeneration: design and preliminary clinical results of the Lipshitz macular implant. J Cataract Refract Surg. 2008; 34(1):87-94.

23. Ahuja AK, Dorn JD, Caspi A, et al; Argus II Study Group. Blind subjects implanted with the Argus II retinal prosthesis are able to improve performance in a spatial-motor task. Br J Ophthalmol. 2011;95(4): 539-543.

24. Alio JL, Mulet EM, Jose M, Ruiz-Moreno, Sanchez MJ, Galal A. Intraocular telescopic lens evaluation in patients with age-related macular degeneration. J Cataract Refract Surg. 2004;30(6):1177-1189.

25. Implantable Miniature Telescope Professional Use Information, FDA-approved product labeling. Retrieved September 29, 2011 Available from: http://www.centrasight.com/pdf/ProfessionalUse2010. pdf. Accessed October 27, 2011.

26. Second Sight to Double Patient Enrollment for Argus II Retinal Implant US. Clinical Trial. Second Sight Medical Products, Inc. May 4, 2009. Web. August 1, 2011. Available from: http://www.2-sight.com/ SSMP_ARVO_2009.pdf. Accessed October 27, 2011

27. "System Overview." Retrieved August 30, 2011. Available from: http://www.2-sight.eu/ee/system-overview. Accessed October 27, 2011.

28. Graf HG, Harendt C, Engelhardt T, et al. High dynamic range CMOS imager technologies for biomedical applications. IEEE Journal of Solid-State Circuits. 2009;44(1):281-289.

29. Brown GC, Brown MM, Lieske HB, Lieske PA, Brown KS, Lane SS. Comparative effectiveness and cost-effectiveness of the implantable miniature telescope. Ophthalmology. 2011;118(9):1834-1843.
Clinical Ophthalmology

\section{Publish your work in this journal}

Clinical Ophthalmology is an international, peer-reviewed journal covering all subspecialties within ophthalmology. Key topics include: Optometry; Visual science; Pharmacology and drug therapy in eye diseases; Basic Sciences; Primary and Secondary eye care; Patient Safety and Quality of Care Improvements. This journal is indexed on

\section{Dovepress}

PubMed Central and CAS, and is the official journal of The Society of Clinical Ophthalmology (SCO). The manuscript management system is completely online and includes a very quick and fair peer-review system, which is all easy to use. Visit http://www.dovepress.com/ testimonials.php to read real quotes from published authors. 